\title{
Model of Multi-Source Nanonetworks for the Detection of BRCA1 DNA Alterations Based on LSPR Phenomenon
}

\author{
Renato Iovine ${ }^{1}$, Valeria Loscrí ${ }^{2}$, Sara Pizzi ${ }^{3}$, Richard Tarparelli ${ }^{1}$, Anna Maria Vegni ${ }^{1}$ \\ ${ }^{1}$ Department of Engineering, University of Roma Tre, Rome, Italy \\ ${ }^{2}$ Dipartimento di Ingegneria Informatica, Modellistica (DIMES), Elettronica e Sistemistica, University of Calabria, Cosenza, Italy \\ ${ }^{3}$ Dipartimento di Ingegneria dell'Informazione, delle Infrastrutture e dell'Energia Sostenibile (DIIES), Mediterranea University of \\ Reggio Calabria, Reggio Calabria, Italy \\ Email: annamaria.vegni@uniroma3.it,renato.iovine@uniroma3.it, richard.tarparelli@uniroma3.it, \\ vloscri@deis.unical.it, sara.pizzi@unirc.it
}

Received July 14, 2013; revised September 2, 2013; accepted September 13, 2013

Copyright (C) 2013 Renato Iovine et al. This is an open access article distributed under the Creative Commons Attribution License, which permits unrestricted use, distribution, and reproduction in any medium, provided the original work is properly cited.

\begin{abstract}
In this paper, we present a multi-source nanonetwork model for biomedical diagnosis applications, based on the Localized Surface Plasmon Resonance by different shape gold nanoparticles (i.e., cylinder, cube, and rod). We present the process of multi-source emission, diffusion, and reception of nanoparticles, based on the ligand/receptor binding. Then, a multi-detection process of DNA alterations is accomplished when nanoparticles are captured at the receiver. The colloidal particles are selectively functionalized with specific splice junctions of gene sequences to reveal simultaneously different alteration that could be associated to an early disease condition. Particularly, full-wave simulations have been carried out for the multi-detection of alternative splice junctions of breast cancer susceptibility gene 1 . The proposed application is verified through numerical results and expressed in terms of Extinction-Cross Section, in the case of synchronous and asynchronous nanoparticles detection. We show that the proposed approach is able to detect DNA alterations, based on a selective nanoparticle reception process.
\end{abstract}

Keywords: Nanoparticles; Nanonetworks; LSPR; DNA Alterations; BRCA1

\section{Introduction}

In the past few decades, nanotechnology has emerged as a novel evolution of technology enabling the design of miniaturized devices at nanoscale level (i.e., nanorobots and nanoparticles). At this scale, the behaviors and characteristics of nanodevices need more comprehension and a deep knowledge with respect to well-known features of devices at the macroscale level [1]. Indeed, a nanodevice is the most basic functional unit, which is allowed to perform very easy tasks, like sensing or actuation, due to the passive nature of these devices. The design and fabrication of nanosensors are difficult tasks for researchers, since a nanosensor is not just a device with reduced size, but has unique properties of nanomaterials and nanoparticles. For instance, nanosensors can detect chemical compounds in concentrations, or the presence of different infectious agents, such as virus or bacteria [2,3]. As an example, in [2] the authors use a Quantum Dots-DNA (QDs-DNA) nanosensor based on Fluorescence Resonance Energy Transfer (FRET) for the de- tection of the target DNA and single mismatch in Hepatitis B Virus (HBV) gene.

Nanoparticles can be designed accordingly to prolong circulation, enhance drug localization [4], increase drug efficacy, and potentially decrease chances of multidrug resistance. Recently, research in the field of cancer diagnostic has made remarkable advances, through the use of nanotechnology for the development of nanoparticles. Basically, gold nanoparticles and NearInfraRed (NIR)-emitting semiconductor QDs are the most widely used. In [5], plasmonic nanoparticles are investigated for sensing applications, while in [6] they are used in several experiments on small animals for in-vivo detection of cancer cells. On the other side, QDs are high fluorescent nanocrystals that can be modified to meet the needs of tumor-selective probes for diagnostic tumor imaging applications, although biocompatibity and toxicity issues can occur [7-9]. In [9], the authors investigate fluorescent imaging with QDs both in vitro and in vivo, and potential barriers to their use in practical 
biomedical applications.

New materials are becoming available for nanoscale design and fabrication of nanodevices. Several physical, chemical and biological nanosensors have been developed by using novel nanomaterials, such as crystal materials. Gallium, phosphate, quartz, and ceramic are chosen for their durability and piezoelectric properties of developing and retaining an electric potential (charge), when subjected to mechanical stress. Graphene and its derivatives, namely Graphene NanoRibbons (GNRs) and Carbon NanoTubes (CNT), are used for the design of nanoantennas [10-12].

Several projects consider the use of nanodevices for medical applications as diagnosis and drug delivery. Among them we cite 1) NAD (Nanoparticles for Therapy and Diagnosis of Alzheimer Disease) whose aim is to utilize nanoparticles specifically engineered for the combined diagnosis and therapy (theranostics) of Alzheimer's disease [13], 2) NANO3T (Biofunctionalized Metal and Magnetic Nanoparticles for Targeted Tumor Therapy) a multi-disciplinary project to develop and explore the use of magnetic nanoparticles as agents for tumor hyperthermia [14], and 3) PANOPTES (Peptidebased Nanoparticles as Ocular Drug Delivery Vehicles) that develops methodology for the manufacture of novel peptide-based nanoparticles for drug delivery [15]. Finally, GRANET project (Graphene-enabled Nanonetworks in the Terahertz Band) investigates the radiation characteristics of planar nano-antennas based on GNRs and larger graphene sheets.

A set of nanodevices, sharing the same medium (e.g., the biological tissue or the bloody flow) and collaborating for the same task, forms a nanonetwork [16]. Nanonetworks are expected to expand the capabilities of single nanodevices and enable new nanotechnology applications in biomedical, environmental, military, industrial and consumer fields.

Communication and signal transmission techniques occurring in nanonetworks are challenging topics, due to the particular environment (i.e., the human body and biological tissues) and to the limited computation skill of nanodevices. Molecular Communication (MC) represents a promising communication paradigm for nanonetworks [16], where molecules (i.e., nanoscale particles) are transmitted by nanomachines, propagate in the medium following a diffusion process, and then arrive at the receiver where a ligand-receptor binding occurs. More in detail, three architectures for nanonetworks are known [17], where for each case nanoparticles can propagate using 1) carrier substances, 2) predictable, and 3) unpredictable (spontaneous) diffusion process i.e., nanoparticles are (are not) subjected to the laws of diffusion in a fluidic medium. The last case where nanoparticles show a behavior totally spontaneous (i.e., a priori un- known) can result as the most challenging.

Recently, the modeling of diffusion-based nanoparticles communications has been investigated in [17] for a point-to-point communication model, where one transmitter emits nanoparticles towards a receiver. The emission, diffusion and reception processes have been described through an analytical model. As a result, this scenario represents the pillar for the theory of the molecular communications, due to the intuitive approach with one transmitter and one receiver.

However, in real scenarios (e.g., for extra-vivo sensing applications), the emission, diffusion and reception processes can show different behaviours. For example, many biomedical applications require a multi-source emission of nanoparticles, where each source (i.e., nanomachine) can emit a particular type of nanoparticles (i.e., with a given shape and size). As a consequence, synchronous and asynchronous nanoparticle emission can affect the diffusion process and degrade network performance (i.e., with an increase of interference and nanoparticles' collisions). The same consideration exists at the receiver side, where a selective reception of nanoparticles occurs (i.e., a given nanoparticle can form a complex only with the "corresponding" receptor).

Other authors have investigated novel schemes of molecular communications, starting from classical concepts of the information theory and adapting into nanonetworks $[18,19]$. In [19], the authors present the MARCO scheme, where the emission process occurs for different kinds of nanoparticles in asynchronous manner, so that information is encoded by means of arrays of nanoparticles.

Once the nanoparticles arrive at the receiver, they are bound and the detection process can occur. Electromagnetic fields and heat are largely used for sensing applications. For example, magnetic nanoparticles can be used to selectively damage or kill cancer cells by heating them, since intracellular hyperthermia has the potential to achieve localized tumor heating without any side effects [20]. Furthermore, by functionalizing the nanoparticles with biological agents such as antibodies or single stranded DNA chains, nanoparticles are forced to bind preferably to specific target cells. This aspect is the focus of our extra-vivo sensing application, that is, the multidetection of DNA alteration of the BReast CAncer susceptibility gene 1 (BRCA1).

BRCA1 and BRCA2 are human genes belonging to a class of genes known as tumor suppressors. Mutation of these genes has been linked to hereditary breast and ovarian cancer. For the BRCA1, this gene causes a genetic susceptibility to breast cancer and changes in its alternative splicing profile have been associated with malignant transformation [21,22]. A woman's risk of developing breast and/or ovarian cancer is greatly increased if she inherits a harmful BRCA1/BRCA2 mutation. 
Genetic tests are available to check for gene mutations. However, due to the vast number of alternative splice variants, tissue and cell type specific pattern of the relative expression levels of these variants, it is difficult to use alternative splicing profiling as a diagnostic tool for breast cancer.

Techniques based on the use of metallic nanoparticles illuminated by an impinging electromagnetic wave (visible and near infrared region) are largely exploited, as alternative approaches for gene alterations detection. The resonant frequency related to the collective electron motion has a strongly dependence on the shape, size, composition of the nanoparticles, as well as on the dielectric properties of the background environment. This technique is known as Localized Surface Plasmon Resonance (LSPR). In this paper, we apply the LSPR phenomenon for the detection of BRCA 1 DNA alteration, when biological gold nanoparticles are captured at the receiver.

The remainder of this paper is organized as follows. In Section 2, we discuss the physical end-to-end model for flows of nanoparticles sent by a transmitter toward a receiver. How nanoparticles are emitted, diffused and received is the objective of this paper. Particularly, we first address the emission process for a multinanomachine source scenario in synchronous and asynchronous mode. Then, we present the diffusion and reception processes. Once the nanoparticles are captured by the receiver, the detection of BRCA1 DNA alterations occurs. The sensing platform is then illustrated in Section 3, while simulation results are described in Section 4. Finally, conclusions are drawn at the end of the paper.

\section{The Physical End-to-End Model for Nanoparticles}

In this section, we will present the physical model of the emission, diffusion and reception of nanoparticles. We will investigate how nanoparticles (1) are transmitted from a set of three nanomachines, (2) diffuse along the gap that separates the transmitter from the receiver, and then (3) arrive at the receiver. All the processes are assumed taking place inside the space $S$, which contains a fluidic medium, initially filled with a homogeneous concentration of particles equal to zero.

In our model, we consider that a single nanoparticle is an indivisible object, which is released to (during the emission process), or collected from (during the reception process), a position in the space $S$, by means of chemical reactions. Moreover, when a nanoparticle is not emitted or received, it is subjected to the diffusion process and moves into the space following the laws of diffusion of particles in a flow. The space is assumed as having infinite extent in any direction, and nanoparticle are free to move everywhere in the space, following a
Random Mobility Model (RMM).

Nanoparticles are emitted by three nanomachines (sources), each of them transmitting nanoparticles of different shape (i.e., cylinder, cube and rod) and same material (i.e., gold). The nanomachines have an independent behavior, such as the $i$-th nanomachine can emit a given flow of nanoparticles in the time instant $t_{i}$, with $i=\{1,2,3\}$. For sensing applications, we assume that the nanomachines can emit nanoparticles both in synchronous (i.e., with $t_{1}=t_{2}=t_{3}$ ), and asynchronous (i.e., $t_{1} \neq t_{2} \neq t_{3}$ ) mode.

A group of nanomachines forms what we define transmitter i.e., an entity emitting different kinds of nanoparticles at different rates and for positive concentrations. We assume the transmitter is placed in the space $S$ in the position along $x$ axis (i.e., at $\left.x_{T}=0 \quad[\mathrm{~nm}]\right)$; the nanoparticles diffuse in the space and reach the receiver laying in the position $x_{R}$, with $x_{R}>x_{T}$ [nm]. In our model, the receiver is comprised of a set of unit cells, each of them has three square gold patches (receptors) deposited on a silica substrate, and functionalized for the capture of target BRCA1 DNA sequences i.e., Target Sequences. Each patch is functionalized with the structure of the BRCA 1 splice with the corresponding sandwich assays. The chemical receptors are BRCA 1 alternative splice variants i.e., $\Delta(5 q, 6), \Delta(9,10)$, and $\Delta(11 q)$ [23]. They are accordingly functionalized for the capture of one type of nanoparticle (i.e., circular, cylinder and square receptors can capture only rod, cylinder and cube nanoparticles, respectively). As a consequence, the probability of capturing a nanoparticle (i.e., the probability of forming a complex) is equal to $1 / 3$ for each type of nanoparticle, considering that each patch is functionalized with a Capture Sequence. Moreover, for sensing purpose, we assume that each nanoparticle can be only captured by the receptor, and not released; the release process is not investigated in this paper.

The physical end-to-end model can be well explained through the scheme in Figure 1, where we have highlighted the main modules of emission, diffusion and reception of nanoparticles. Notice that each process is trigged by an input signal: an initial signal $s_{T}(t)$ modulates the particle concentration rate at the transmitter side $r_{T}(t)$; this is then propagated by the diffusion process to the receiver side, which finally detects the concentration signal $c_{R}(t)$ and then generates the received signal $s_{R}(t)$.

Each module is represented as a black box (i.e., $\boldsymbol{A}(f), \boldsymbol{B}(f)$, and $\boldsymbol{C}(f))$. Our aim is the modeling of the delay that occurs in 1) the transmitter module i.e., $\tau_{A}(f)$ [s] the time necessary to transmit a flow of nanoparticles, 2$)$ the diffusion process i.e., $\tau_{B}(f)$ [s] the time for a nanoparticle to move along the gap from 


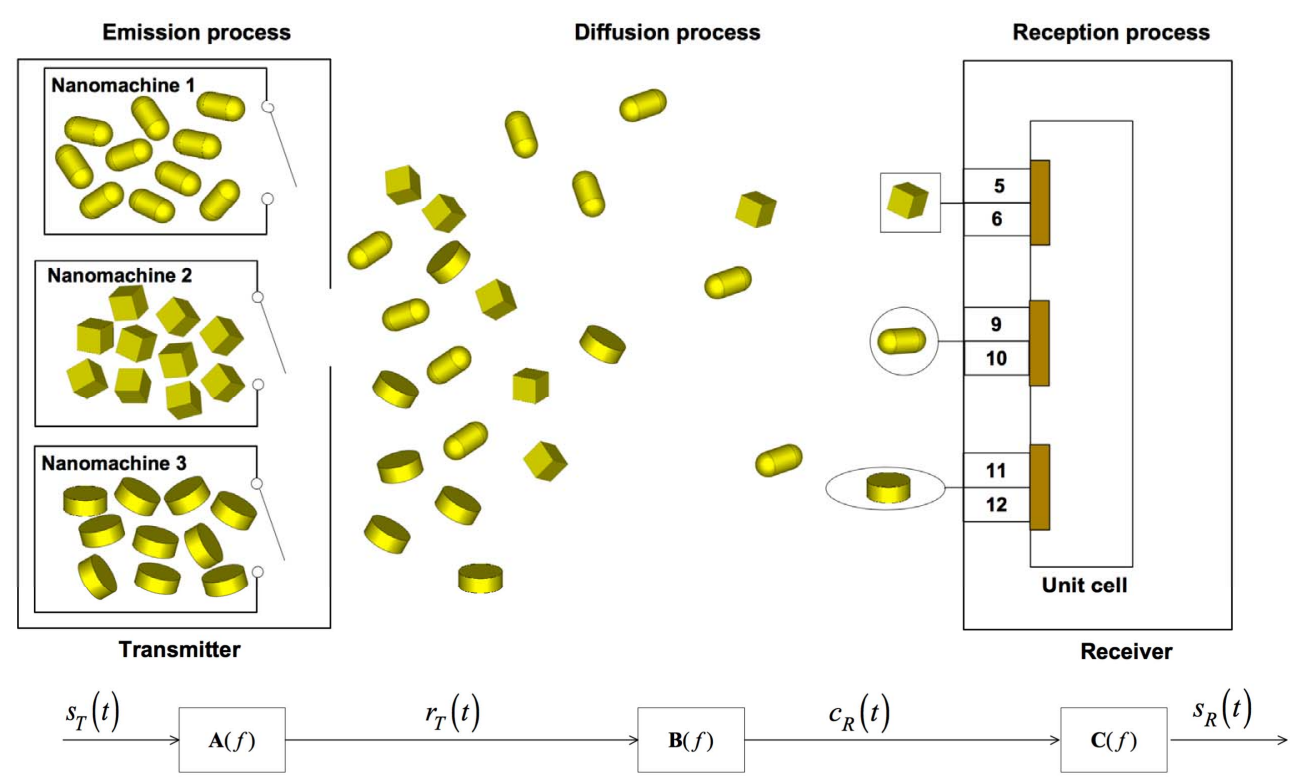

Figure 1. End-to-end physical model of the nanonetwork scenario, comprised of one transmitter and one receiver. In the emission process, the transmitter is comprised of 3 nanomachines, allowed to emit nanoparticles (a)synchronously. The diffusion process is ruled according to Fick's first law. In the reception process, nanoparticles are captured in "selective" mode. At the receiver side, we show the unit cell comprised of three gold patches. In each patch, numbers denote different exons, as appear in [24] (they are not drawn to scale).

the transmitter to the receiver, and 3 ) at the receiver i.e., $\tau_{C}(f)[\mathrm{s}]$ the time necessary to receive the nanoparticles. Equations (9), (34) and (39) will be introduced to describe the delay in the transmission, diffusion and reception process, respectively.

\subsection{Multi-Nanomachine Emission Process}

The emission process aims to modulate the particle concentration rate at the transmitter. This is comprised of three nanomachines, each of them is modeled as a box containing a nanoparticle concentration value $c_{i, \text { in }}$, with $i=\{1,2,3\}$, and provided with an aperture from where the nanoparticles can exit, when emitted.

Each nanomachine receives the same input signal $s_{T}(t)$, which represents the input of the end-to-end model. The particle flux is defined as the net particle concentration leaving/entering the transmitter per unit time. The particle concentration at the output of the $i$-th nanomachine $c_{i, \text { out }}$, with $i=\{1,2,3\}$, is the average concentration in the proximity of the $i$-th nanomachine, assumed equal to zero initially. This variation in the particle concentration at the output of the transmitter is expressed as:

$$
r_{T}(t)=\sum_{i} \frac{\mathrm{d} c_{i, \text { out }}(t)}{\mathrm{d} t}
$$

where we assumed the superposition principle occurs.

The particle concentration flux emitted by the $i$-th nanomachine is stimulated by a concentration gradient between $c_{i, \text { out }}$ and $c_{i \text {,in }}$. Basically, the particle concen- tration inside each nanomachine is trigged according to the input signal $s_{T}(t)$ : for increases (decreases) of $s_{T}(t)$, the transmitter increments (reduces) each $c_{i, \text { in }}$, and then the $i$-th particle concentration at the output $c_{i, \text { out }}$ is increased (decreased) too; as a result, the expression in (1) increases (decreases), as well. It is worth noticing that a negative rate modulation can occur when $c_{i, \text { in }}<c_{i, \text { out }}$, and then $r_{T}(t)$ is negative. However, this case is not assumed in our model i.e., the nanomachines emit flows of nanoparticles.

This behavior can be well depicted through an electrical parallel RC circuit, as that one shown in Figure 2(a). We consider the $i$-th nanomachine as the parallel group of the resistance $R_{i}$ and capacitor $C_{i}$, with $i=\{1,2,3\}$. The $i$-th switch is closed when the $i$-the nanomachine is emitting nanoparticles, otherwise it is open. Multi-transmissions are allowed in synchronous and asynchronous mode. In the case of $i=3$ nanomachines transmitting at the same time instant, the circuit of Figure 2(a) becomes as in Figure 2(b), where the input current $I_{\text {in }}(t)$ represents the input signal $s_{T}(t)$ of the end-to-end model of Figure 1, while the output current $I_{\text {out }}(t)$ represents the output signal $r_{T}(t)$.

The resistor $R_{\|}$and the capacitor $C_{\|}$are obtained as follows:

$$
R_{\|}=\frac{R_{1} R_{2} R_{3}}{R_{2} R_{3}+R_{1} R_{3}+R_{1} R_{2}}, \quad C_{\|}=C_{1}+C_{2}+C_{3} .
$$

From the electrical circuit theory, we compute the Time Fast Fourier Transform (TFFT) of the equivalent 


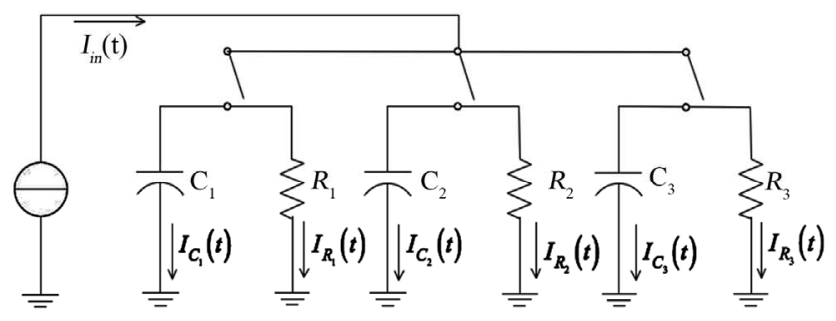

(a)

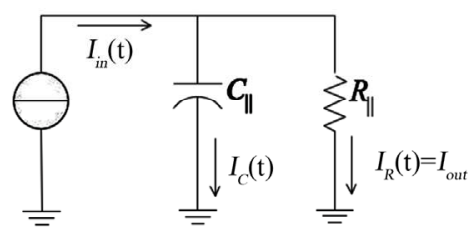

(b)

Figure 2. (a) Multi-nanomachine emission process circuit model, where each RC group represents a transmitting nanomachine, and (b) equivalent circuit model.

RC circuit of Figure 2(b), that is

$$
\boldsymbol{H}_{R C}(f)=\frac{\tilde{\boldsymbol{V}}_{\text {out }}(f)}{\tilde{\boldsymbol{V}}_{\text {in }}(f)}=\frac{1}{1+j \omega R_{\|} C_{\|}},
$$

where $\tilde{V}_{\text {out }}(f)$ and $\tilde{V}_{\text {in }}(f)$ are the Fourier transforms of the input and output voltage, respectively.

Notice that the nanoparticle concentration rate $r_{T}(t)$ can be identified with the particle concentration flux $J_{T}(x, t)$ at the output of the transmitter i.e., for $x=x_{T}$. The particle concentration flux $J_{T}(x, t)$ is dependent on the particle concentration gradient at time $t$ and position $x$ through the Fick's first law, as follows

$$
J_{T}(x, t)=-D \nabla c(x, t),
$$

where $D\left[\mathrm{~cm}^{2} / \mathrm{s}\right]$ is the diffusion coefficient, assumed as a constant value for a given fluidic medium. The diffusion coefficient depends on size and shape of nanoparticles, as well as the interaction with the solvent and viscosity of solvent. In (4), the nanoparticle concentration flux $J_{T}(\bar{x}, t)$ can be related to the output current $I_{\text {out }}$, flowing along the resistor of the circuit in Figure 2(b), while the particle concentration gradient corresponds to the voltage along the capacitor $C_{\|}$, such as:

$$
J_{T}(x, t)=I_{\text {out }}, \quad-\nabla c(x, t)=V_{C_{\|}} .
$$

From the circuit theory, we have

$V_{C_{\|}}=R_{\|} I_{\text {out }}$, (6)

and then it follows

$$
J_{T}(x, t)=\frac{-\nabla c(x, t)}{R_{\|}} \Rightarrow R_{\|}=\frac{1}{D} .
$$

This shows that the diffusion coefficient $D$ takes into account the contributions of three nanoparticle flows. Now, we can consider the TFFT $\tilde{\boldsymbol{A}}(f)$ of the transmitter module be equivalent to (3), that is the TFFT $\boldsymbol{H}_{R C}(f)$ of the RC circuit in Figure 2(b)

$$
\tilde{\boldsymbol{A}}(f)=\boldsymbol{H}_{R C}(f) \text {, }
$$

from which we can evaluate the delay $\tau_{A}(f)$ for the transmitter module, as:

$$
\tau_{A}(f)=-\frac{\mathrm{d} \phi_{A}(f)}{\mathrm{d} f},
$$

where $\phi_{A}(f)$ is the phase of the TFFT of $\tilde{\boldsymbol{A}}(f)$, whose expression is easily computed as

$$
\phi_{\boldsymbol{A}}(f)=\arctan \left(\frac{\operatorname{Im}[\tilde{\boldsymbol{A}}(f)]}{\operatorname{Re}[\tilde{\boldsymbol{A}}(f)]}\right)=\arctan \left(-\omega R_{\|} C_{\|}\right) .
$$

\subsection{Nanoparticle Diffusion Process}

The diffusion process of nanoparticles in the space $S$ is strictly related to 1$)$ the concentration rate $r_{T}(t)$, that is the output from the emission process, and 2) the way the particle diffuse in a medium i.e., in our case it is a fluid medium.

In the proposed model, we assume gold particles with different geometries that are similar to each other in terms of volume occupancy. This assumption makes easier the analysis of the diffusion process. It is well known that diffusion is always along a chemical potential gradient and stops when chemical potentials are constant along the space [24]. Typically, the chemical potential increases when concentration increases too and, for this reason, it is a fair assumption to express the diffusion in terms of concentration.

In order to understand how nanoparticles move in a three-dimensional space (lattice), we consider $c(x)$, and $\mu(x)$, as the concentration and the chemical potential at position $x$ in the space $S$, respectively. Then, in position $(x+a)$, where $a$ represents a space deviation, that is the step, we have:

$c(x+a)=c(x)+\frac{a \mathrm{~d} c}{\mathrm{~d} x}, \quad \mu(x+a)=\mu(x)+\frac{a \mathrm{~d} \mu(x)}{\mathrm{d} x}$,

Let $\Delta G^{A}[\mathrm{~kJ} / \mathrm{mol}]$ be the activation barrier i.e., the minimum energy required to start a chemical reaction; this also means that for a chemical reaction to proceed at a reasonable rate, there should exist an appreciable number of molecules with energy equal to or greater than the activation barrier. Then, we can define the forward rate as

$$
\propto \mathrm{e}^{-\Delta G^{\mathrm{A}} / R T},
$$


where $R$ is the universal gas constant, $A$ is a constant, and $T[K]$ is the temperature. We assume that in our system there is no meaningful change in terms of temperature; in fact, as a specific case, we can consider an extra-vivo application (e.g., a hair sample), and then such assumption is plausible. Moreover, (12) is usually true with the assumption that $\Delta G \ll R T$, where the free energy change is small within short distances. This allows writing the following:

$$
\Delta G=G(x+a)-G(x)=\mu(x+a)-\mu(x),
$$

and also

$$
\frac{\Delta G}{R T}=\frac{\mu(x+a)}{R T}-\frac{\mu(x)}{R T}=\frac{a}{R T} \frac{\mathrm{d} \mu}{\mathrm{d} x} .
$$

Let us introduce $v$ as the vibrational frequency. Then, a nanoparticle will have enough energy per second to overcome the activation barrier $\left(v \mathrm{e}^{-\triangle G^{A} / R T}\right)$ times. In practice, we can define $\Gamma$ as the number of jumps of step $A$ per second, such as:

$$
\Gamma=v \mathrm{e}^{-\Delta G^{A} / R T} .
$$

Since we are considering a three-dimensional space, modeled as a lattice, the nanoparticles are free to move along six equivalent directions of vectors $\hat{\boldsymbol{x}}, \hat{\boldsymbol{y}}$, and $\hat{\boldsymbol{z}}$ (i.e., $\pm x, \pm y$, and $\pm z$, respectively). This means that the number of jumps along the $i$-th direction in the time unit [s] is

$$
\Gamma_{\hat{i}}=\frac{v}{6} \mathrm{e}^{-\Delta G^{A} / R T},
$$

where $\hat{\boldsymbol{i}}=\{\hat{\boldsymbol{x}}, \hat{\boldsymbol{y}}, \hat{\mathbf{z}}\}$. We can now calculate $R_{\hat{i}}$ as the net forward rate along the direction $\hat{\boldsymbol{i}}$ :

$$
R_{\hat{i}}=\Gamma_{\hat{i}} \cdot \frac{-\Delta G}{R T}=\frac{v}{6} \mathrm{e}^{-\Delta G^{\mathrm{A}} / R T}\left(\frac{-a}{R T} \frac{\mathrm{d} \mu}{\mathrm{d} x}\right),
$$

where $\mathrm{d} \mu / \mathrm{d} x<0$.

Let us consider the nanoparticles move along the direction of $(\hat{\boldsymbol{x}}, 0,0)$. Considering $n(x)$ as the number of nanoparticles per unit area in position $x$, we can compute the number of nanoparticles passing through the unit area in the unit time along $\hat{\boldsymbol{x}}$ as:

$$
J_{\hat{x}}=n(x) \cdot R_{\hat{x}}=n(x) \cdot \frac{v}{6} \mathrm{e}^{-\Delta G^{A} / R T}\left(\frac{-a}{R T} \frac{\mathrm{d} \mu}{\mathrm{d} x}\right) .
$$

Since $n(x)=a \cdot c(x),(18)$ can be written as:

$$
J_{\hat{x}}=-a^{2} \frac{v}{6} \mathrm{e}^{-\Delta G^{A} / R T} \cdot\left(\frac{c(x)}{R T} \frac{\mathrm{d} \mu}{\mathrm{d} x}\right) .
$$

Now, let us consider the expression of chemical potential $\mu(x)$ as

$$
\mu(x)=\mu^{0}+R T \ln (a(x))=\mu^{0}+R T \ln (\gamma c(x)),
$$

where $a(x)$ is the activity of the particle in position $x$, and $\gamma$ is a constant, named activity coefficient. Then, the derivative of (20) is:

$$
\frac{\mathrm{d} \mu}{\mathrm{d} x}=\frac{R T}{c(x)} \frac{\mathrm{d} c(x)}{\mathrm{d} x},
$$

and by substituting (21) into (19), we will have:

$$
J_{\hat{x}}=-a^{2} \frac{v}{6} \mathrm{e}^{-\Delta G^{A} / R T} \frac{\mathrm{d} c(x)}{\mathrm{d} x},
$$

and given the similarity between (4) and (22), we can calculate the intrinsic diffusion coefficient $D\left[\mathrm{~cm}^{2} / \mathrm{s}\right]$ as:

$$
D=a^{2} \frac{v}{6} \mathrm{e}^{-\frac{\Delta G^{A}}{R T}} .
$$

By considering the diffusion process in a threedimensional space, where a nanoparticle can move following a Random Mobility Model, along six equivalent directions, we obtain:

$$
D=\frac{1}{6} \Gamma a^{2}=a^{2} \frac{v}{6} \mathrm{e}^{-\Delta G^{A} / R T},
$$

from which we can recompute (15) as:

$$
\Gamma=v \mathrm{e}^{-\frac{\Delta G^{A}}{R T}}=\frac{6 D}{a^{2}} .
$$

Unfortunately, the Fick's first law works when applied to steady state systems, that is the concentration will keep constant in the time and along the space. In the specific application we are considering, the concentration can change in the time, since our nanoparticle diffuse along the space by determining different levels of concentration. For this reason, we need to consider the Fick's second law.

Let us define $c(x, t)$ and $J(x)$ as, respectively, the local concentration and the diffusion flux through an unit area $A$ in position $x$. Then we can write:

$$
\mathrm{d} c(x)=\frac{[J(x)-J(x+\mathrm{d} x)] \mathrm{d} t A}{A \mathrm{~d} x},
$$

and

$$
J(x+\mathrm{d} x)=J(x)+\frac{\mathrm{d} J}{\mathrm{~d} x} \mathrm{~d} x,
$$

from which it follows:

$$
\frac{\mathrm{d} c(x, t)}{\mathrm{d} t}=-\frac{\mathrm{d} J}{\mathrm{~d} x},
$$

and by substituting $\mathrm{d}$ with $\delta,(28)$ is equivalent to

$$
\frac{\delta c(x, t)}{\delta t}=-\frac{\delta J}{\delta x} \text {. }
$$


Thus, from the first Fick's law, we can obtain:

$$
\frac{\delta c(x, t)}{\delta t}=-\frac{\delta J}{\delta x}=D \frac{\delta^{2} c}{\delta x^{2}},
$$

and for a three-dimensional space we can rewrite (30) as:

$$
\frac{\delta c(x, t)}{\delta t}=D \Delta^{2} c(x, t) .
$$

It is worth to notice that at the equilibrium state (i.e., when the concentration does not change), we obtain the first Fick's law, that can be considered as a specific case of the second law, when applied to a steady state. However, as outlined in [17] and [25], the second Fick's law is in contradiction with the theory of special relativity. In practice, the second Fick's law states for an instantaneous propagation of particles information, that is equivalent to allow a super-luminal information propagation speed. The Telegraph Equation [26] is commonly used to fix this problem, by considering an additional term $r(x, t)$ that represents the incoming concentration rate:

$$
\tau_{d} \frac{\delta^{2} c(x, t)}{\delta t^{2}}+\frac{\delta c(x, t)}{\delta t}=D \Delta^{2} c(x, t)+r(x, t),
$$

where the term $\tau_{d}$ is the relaxation time and is related with the heat diffusion process. In fact, the Telegraph Equation was originally formulated for the case of heat transfer, but it can also be applied to the diffusion of particles, as in our case.

For a random walk in a three-dimension space, after $n$ steps a nanoparticle travels an average distance of $\sqrt{n} \cdot a$, where a step is the time necessary for one jump [s], and the jump length is $a[\mathrm{~m}]$. Thus, after a time $t$ $[\mathrm{s}]$, the nanoparticle jumps $\Gamma t$ times and moves a distance of

$$
r=a \sqrt{\Gamma t}=a \sqrt{\frac{6 D t}{a^{2}}}=\sqrt{6 D t} \approx 2.4 \sqrt{D t},
$$

where $r[\mathrm{~nm}]$ is defined as the diffusion length. This represents the average space a nanoparticle moves during the diffusion process, depicted as a black box in Figure 1.

From (33) we obtain $\tau_{B}(f)$ [s] that is the TFFT of the delay that a nanoparticle experiences to move along the gap from the transmitter to the receiver:

$$
\tau_{B}(f)=R(f) * \frac{1}{V_{\text {fluid }}(f)},
$$

where $R(f)$ is the TFFT of $r, V_{\text {fluid }}(f)$ is the TFFT of $v_{\text {fluid }}[\mathrm{mm} / \mathrm{s}]$, that is the velocity of the fluid where the nanoparticles diffuse, and $*$ is the convolutional operator. In accordance to Equation (8) in [27], the velocity of nanoparticles $v_{\text {nanoparticle }}$ is equal to the fluid velocity plus an additional term $\Omega$ that takes into account the geometry of the nanoparticle, and the collisions with other molecules, that is:

$$
v_{\text {nanoparticle }}=v_{\text {fluid }}+\Omega \text {. }
$$

In our specific scenario i.e., an extra-vivo application, the nanoparticles move into a fluid solution that is not constrained in a vessel (e.g., the bloody flow). Then, we can neglect the additional term $\Omega$, and consider the nanoparticles velocity approximates that of the fluid.

\subsection{Nanoparticle Reception Process}

In this subsection we address the nanoparticle reception process. However, the structure of the receiver is presented in Section 3, where the geometry and electromagnetic properties are described.

The reception process has the task to sense the particle concentration at the receiver and to produce, accordingly, the output signal $s_{R}(t)$, which represents the output of the end-to-end model. The nanoparticle reception is realized by means of chemical receptors that homogeneously occupy the reception space $S_{r}$, with $S_{r} \subset S$, where the receiver lays. Receptors take place only in correspondence of BRCA 1 alternative splice variants and the output signal $s_{R}(t)$ is related to the number of obtained complexes, over the total number of available chemical receptors.

Similar to the transmission process, the behavior of the reception process can be well depicted through the electrical RC circuit shown in Figure 3(a). We consider the $i$-th chemical receptor as the serial group of the resistance $R_{i}$ and capacitor $C_{i}$, with $i=\{1,2,3\}$ (i.e., a maximum number of three complexes can take place within the receiver unit cell). The capacitor value $C_{i}$ can be considered equal to the number of receptors, while the resistor value $R_{i}$ is inversely proportional to the probability of capturing a nanoparticle (i.e., forming a complex) [17]. The $i$-th switch is close when the $i$-th chemical receptor is involved in a complex, otherwise it is open. In the case that $i=3$ complexes are established at the same time instant, the circuit of Figure 3(a) becomes as in Figurr 3(b), where the input voltage $V_{\text {in }}(t)$ represents the particle concentration $C_{R}(t)$ at the receiver, while the output current $I_{\text {out }}(t)$ represents the system output signal $s_{R}(t)$ of the end-to-end model (see Figure 1).

The resistor $R_{R x}$ and the capacitor $C_{R x}$ are related, respectively, to the real and imaginary part of the following impedance:

$$
\begin{aligned}
Z_{R x} & =R_{R x}+\frac{1}{j \omega C_{R x}} \\
& =\frac{1}{\left(\frac{j \omega C_{1}}{1+j \omega C_{1} R_{1}}\right)+\left(\frac{j \omega C_{2}}{1+j \omega C_{2} R_{2}}\right)+\left(\frac{j \omega C_{3}}{1+j \omega C_{3} R_{3}}\right)}
\end{aligned}
$$



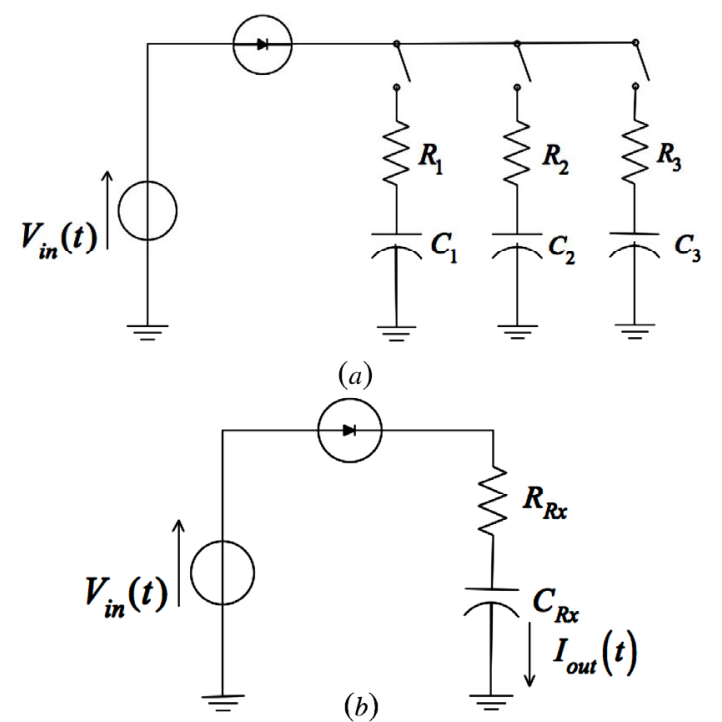

(b)

Figure 3. (a) Multi-nanoparticle reception process circuit model, where each RC series represents the reception of one kind of nanoparticle flow (i.e., cube, rod, and cylinder), and (b) equivalent circuit model.

From the electrical circuit theory, we compute the TFFT of the equivalent RC circuit of Figure 3(b) as follows:

$$
\boldsymbol{H}_{R C}(f)=\frac{\tilde{\boldsymbol{I}}_{\mathrm{out}}(f)}{\tilde{\boldsymbol{V}}_{\text {in }}(f)}=\frac{j \omega C_{R x}}{1+j \omega R_{R x} C_{R x}},
$$

where $\tilde{\boldsymbol{I}}_{\text {out }}(f)$ and $\tilde{\boldsymbol{V}}_{\text {in }}(f)$ are the Fourier transforms of the output current and the input voltage, respectively. It follows that we can consider the TFFT $\tilde{\boldsymbol{C}}(f)$ of the receiver module be equivalent to (37):

$$
\tilde{\boldsymbol{C}}(f)=\boldsymbol{H}_{R C}(f),
$$

from which we can evaluate the delay $\tau_{C}(f)$ for the receiver module, as:

$$
\tau_{C}(f)=-\frac{\mathrm{d} \phi_{C}(f)}{\mathrm{d} f},
$$

where $\phi_{C}(f)$ is the phase of the TFFT of $\tilde{\boldsymbol{C}}(f)$, whose expression is easily computed as:

$$
\phi_{C}(f)=\arctan \left(\frac{\operatorname{Im}[\tilde{\boldsymbol{C}}(f)]}{\operatorname{Re}[\tilde{\boldsymbol{C}}(f)]}\right)=\arctan \left(\frac{1}{\omega R_{R x} C_{R x}}\right) .
$$

\section{Sensing Platform}

In this section we analyze the sensing platform able to reveal DNA multi-alterations of the BRCA1 gene based on the LSPR phenomenon. LSPR occurs when an electromagnetic plane wave impinges on metallic nanoparticles that are electrically small. In this condition the free electrons of the nanoparticle follow collectively the electromagnetic oscillations. Therefore, in order to study the electromagnetic behavior of nanoparticle, a quasistatic approximation can be carried out.

It is well known that in case of an arbitrary shaped nanoparticle, the dyadic polarizability $\underline{\alpha}$, can be expressed as:

$$
\underline{\underline{\alpha}}=V \varepsilon_{e} \sum_{m=1}^{3} \frac{\varepsilon_{i}-\varepsilon_{e}}{\varepsilon_{e}+L_{m}\left(\varepsilon_{i}-\varepsilon_{e}\right)} \underline{u}_{m} \underline{u}_{m},
$$

where $V$ is the particle volume, $\varepsilon_{e}$ is the dielectric permittivity of the surrounding environment, $\varepsilon_{i}$ is the complex dielectric permittivity of the metallic nanoparticle, $\underline{u}_{m}$ are unit vectors in the direction of the principal axes of the particle, $L_{m}$ are the three components of the corresponding depolarization dyadic, that is

$$
\underline{\underline{L}}=L_{1} \underline{u}_{1} \underline{u}_{1}+L_{2} \underline{u}_{2} \underline{u}_{2}+L_{3} \underline{u}_{3} \underline{u}_{3} \text {. }
$$

Therefore, the resonant frequency of the electron motion strongly depends on the nanoparticle size, shape, composition, and surrounding dielectric environment [28].

By using the same procedure conducted in [29], it is possible to design analytical models describing the extinction cross-section properties for different nanoparticle shapes. The general corresponding expression can be written as the sum of absorption and scattering phenomenon, that is

$$
C_{e x t}=k \cdot \operatorname{Im}[\alpha]+\frac{k^{4}}{6 \pi}|\alpha|^{2},
$$

where $k=\frac{2 \pi n}{\lambda}$ is the wavenumber, $\lambda$ is the

wavelength, and $n=\sqrt{\varepsilon_{e}}$ is the refractive index of the surrounding dielectric environment.

To design the sensing platform different nanoparticles are considered. The use of different nanoparticles shapes ensure the possibility to tune the electromagnetic response of each particle in a different frequencies range. According to [5], in this study different gold particles (i.e. cube, rod, and elliptical cylinder) have been chosen. Considering such elements, the corresponding scalar components of the depolarization factor for the principal nanoparticle axis i.e., $L_{\text {cubel }}, L_{\text {rod1 }}$, and $L_{\text {ell.cyl.1 }}$, are respectively:

$$
\begin{aligned}
& L_{\text {cube } 1}=\frac{10 \sqrt{2}\left(1-\frac{1}{\sqrt{2}}\right) \pi}{l}, \\
& L_{\mathrm{rod} 1}=1-\frac{1}{\sqrt{1+\frac{a_{r}^{2}}{\left(a_{r}+\frac{h}{4}\right)^{2}}}},
\end{aligned}
$$




$$
L_{\text {ell.cyl.1 }}=\frac{1}{\pi}\left(1-\frac{h}{4 \sqrt{a^{2}+\frac{h^{2}}{16}}}\right) \cdot E\left[\frac{1}{1-\left(1-\frac{b^{2}}{a^{2}}\right)^{2}}-1\right],
$$

where $E[\cdot]$ is the Complete Elliptic Integral of the second kind, $l[\mathrm{~nm}]$ is the cube side length, $a[\mathrm{~nm}]$ and $b \quad[\mathrm{~nm}]$ are the elliptical cylinder base semi-axes lengths, $a_{r}[\mathrm{~nm}]$ is the rod thickness, and $h[\mathrm{~nm}]$ is the height length of the whole.

In order to provide the multi-detection of DNA alterations, we have designed the geometrical parameters of such particles in order to resonate at different frequencies. By using the aforementioned expressions we have assumed the following geometrical parameters for the selected particles: (1) the nanocube with $l=51 \mathrm{~nm}$, (2) the nanorod with $h=64 \mathrm{~nm}$ and with $a_{r}=9 \mathrm{~nm}$, and (3) the elliptical cylinder nanoparticle with $a=35$ $\mathrm{nm}$ and $b=7 \mathrm{~nm}$ and with $h=10 \mathrm{~nm}$. In this condition the cube, rod and elliptical cylinder nanoparticles resonate at $542 \mathrm{~nm}, 671 \mathrm{~nm}$ and $808 \mathrm{~nm}$ respectively. For such nanoparticles configurations, we will show how the multi-detection condition for DNA alterations can be established.

It is well know that various shapes of nanoparticles can be functionalized with single DNA sequences [30,31]. In this paper we consider the nanoparticles functionalized with the corresponding DNA Probe Sequence (PS) of alternative splicing junctions of BRCA1, as shown in Figure 4. Notice that in the nanocube the $\mathrm{PS}_{1}$ has been putted in each face, while for the nanorod and the elliptical cylinder, the $\mathrm{PS}_{2}$ and $\mathrm{PS}_{3}$ lay on the top and bottom of the particles. The mean derives from the peculiar metallic nanoparticles properties. With such configurations, the nanocube can bind in six positions, while for the rod and elliptical cylinder only two positions are allowed.

The three corresponding DNA Capture Sequences (CS) of alternative splicing junctions of BRCA1 are allocated on three square gold patches deposited on silica substrate as shown in Figure 5. The geometry of the silica substrate has been chosen for $a=600, b=200, c=50$, and $d=150 \quad[\mathrm{~nm}]$. A sandwich structure is obtained, whenever the corresponding Target Sequences (TSs) of alternative splice variants of BRCA1 are present (Figure 6).

In the next section, we will show the electromagnetic response when different ligand-receptor bindings are considered.

\section{Simulation Results}

In this section we discuss and analyze the simulation results to verify the ability to detect the multi DNA alterations, particularly for BRCA 1 . In order to test the proposed platform, extensive numerical analysis have been conducted through full-wave simulation [32].

The sensing platform is excited by an impinging plane wave, having the electric field $E$ parallel to the principal axis of the particles. The excitation is employed to analyze the electromagnetic properties, in terms of extinction cross-section. In the simulations we have assumed (1) for gold the experimental values of the complex permittivity reported in [33], (2) for silica a complex refractive index $n=1.44+i 0$ according to [34] and (3) an in silico replica of BRCA1 alternative splice variant as reported in [23].

We considered two cases of nanoparticle detection i.e., the synchronous and asynchronous cases. In the first case, just one nanomachine has transmitted a flow of nanoparticles, and so we have just one kind of ligandreceptor bind (e.g., the nanomachine 2 has transmitted a flow of nanocubes), while in the synchronous case, all

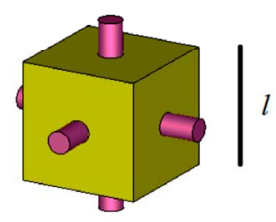

(a)

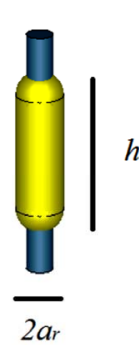

(b)

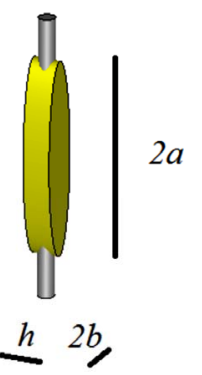

(c)

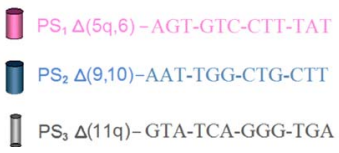

Figure 4. Schematic model of (a) cube, (b) rod, and (c) elliptical cylinder nanoparticle

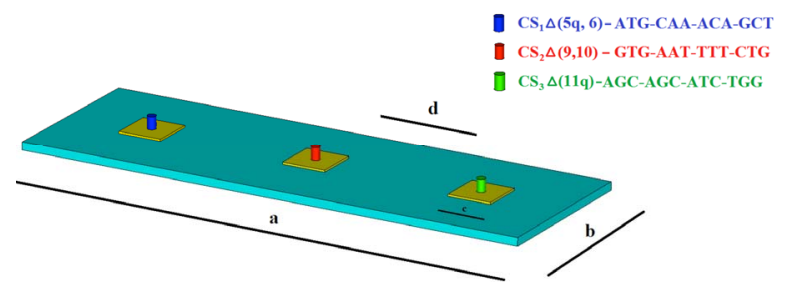

Figure 5. Unit cell of the sensing platform, comprised of three square gold patches, functionalized with three different Capture Sequences (depicted in blue, red, and green).

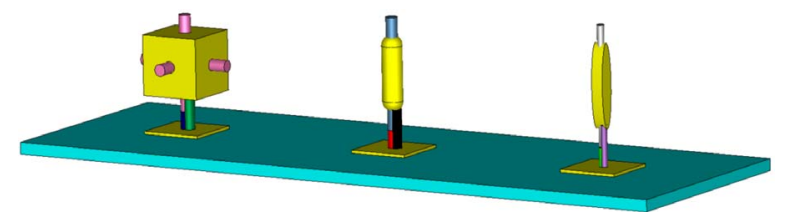

Figure 6. Ligand-receptor binding of three nanoparticles. Green, black and violet sections represent the Target Sequences for each nanoparticle. 
the nanomachines have transmitted the own nanoparticles.

Figure 7 (a) reported the extinction cross-section spectrum in the case of nanocube binding for the asynchronous case. We notice the peak of the extinction cross-section at $542 \mathrm{~nm}$; this means that $\mathrm{CS}_{1}$ and $\mathrm{PS}_{1}$ are bound. In the case of rod and elliptical cylinder nanoparticles binding, under the assumption of asynchronous transmission, the obtained extinctions crosssection are shown in Figures 7(b) and (c), respectively. In Figure 7(b) we notice the peak of the extinction cross-section at $671 \mathrm{~nm}$ meaning that $\mathrm{CS}_{2}$ and $\mathrm{PS}_{2}$ are bound, while in Figure 7(c) the peak is at $808 \mathrm{~nm}$, when $\mathrm{CS}_{3}$ and $\mathrm{PS}_{3}$ are bound.

As aforementioned, the electromagnetic response obtained from each nanoparticle must be different and independent in terms of resonant wavelength, amplitude and magnitude width, respectively. Figure 7 shows as the sensing platform could be efficiently applied for DNA alterations multi-detection. For each ligand-receptor binding, we notice a typical extinction cross-section which allow the nanoparticles multi-detection process. To demonstrate this ability, in Figure 8 the extinction cross-section is shown, for the case of three nanoparticles bindings. Obviously the sensor is able to recognize also no binding (see the red line in Figure 8).

The synchronous transmission can present other three binding combinations, such as the cases of 1) $\mathrm{TS}_{1}+$ $\mathrm{TS}_{2}$,2) $\mathrm{TS}_{2}+\mathrm{TS}_{3}$, and 3) $\mathrm{TS}_{1}+\mathrm{TS}_{3}$. Due to the geometry of the unit cell, the coupled effects among near nanoparticles are negligible and also each nanoparticle shows near electric field enhancement at the proper resonant wavelength. Figure 9(a) shows the near electric field distribution at the corresponding resonant wavelength of nanocube particle. In this way the sensing platform is able to recognize TS, DNA alteration. Similar results are obtained for $\mathrm{TS}_{2}$ and $\mathrm{TS}_{3}$ DNA alterations. In fact, by observing the near electric field distributions at other specific resonant wavelengths we can see that specific nanoparticle is excited (Figures 9(b) and (c)).

\section{Conclusions}

In this paper, we presented the end-to-end model from the transmittion up to the reception of flows of nanoparticles for biomedical sensing applications. How nanoparticles diffuse is also investigated. The proposed model has been verified for the multi-detection of DNA alterations, specifically for the BRCA1 gene.

In our model, we considered three kinds of metallic nanoparticles (i.e., cube, rod, and cylinder), which can form a complex with chemical receptors. Based on a ligand-receptor binding, it is possible to detect DNA

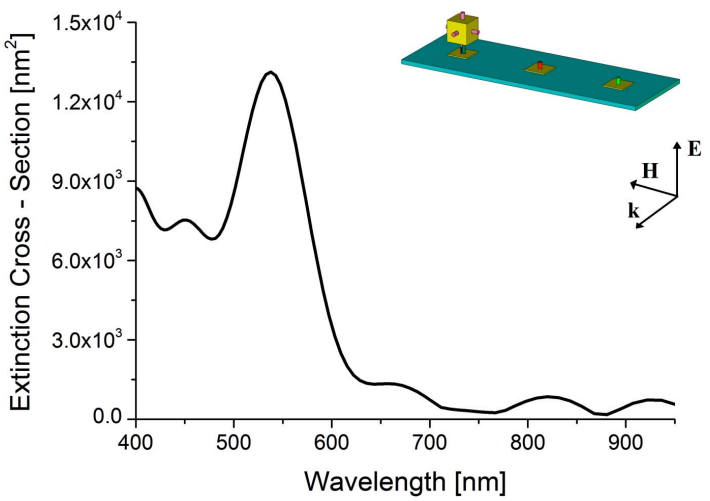

(a)

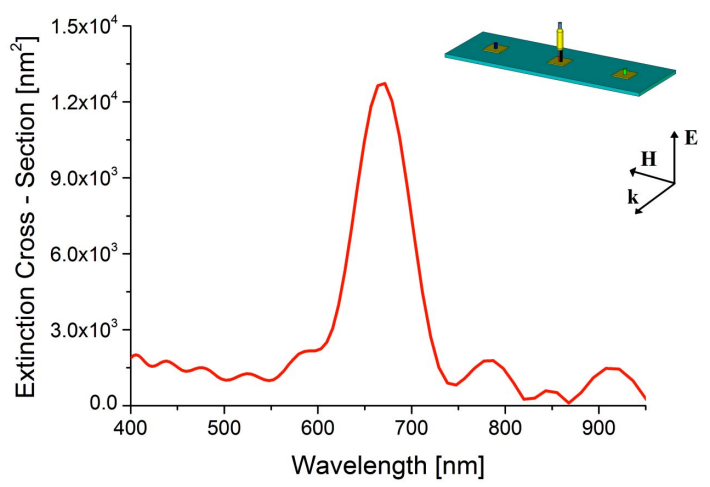

(b)

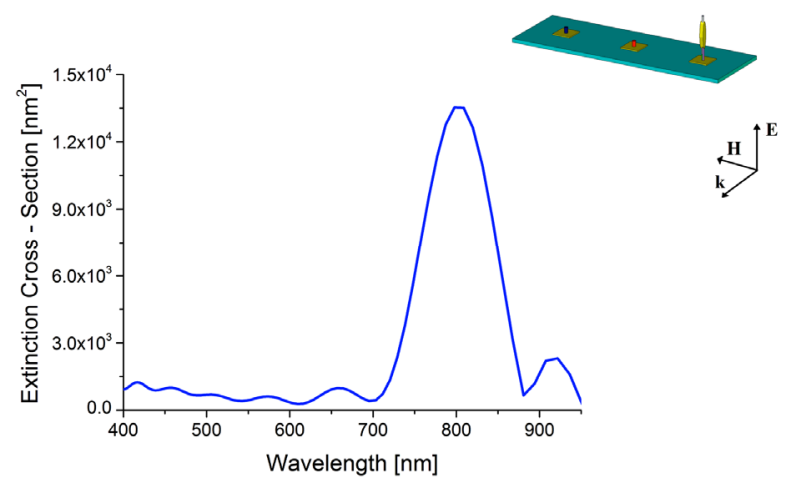

(c)

Figure 7. Asynchronous detection. Extinction Cross-Section spectra obtained for (a) TS1, (b) TS2, and (c) TS3, only.

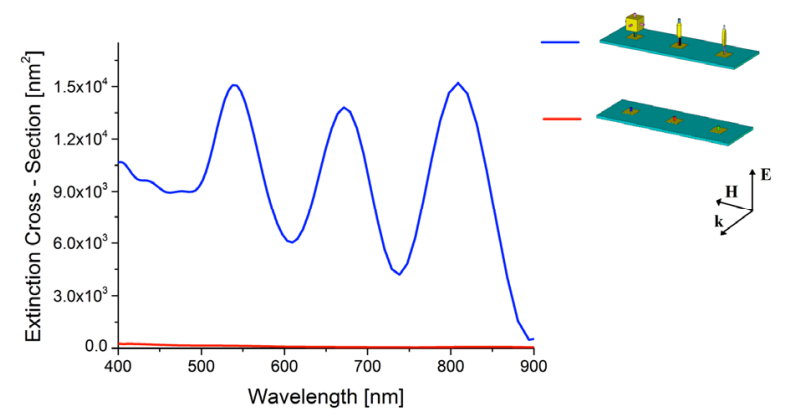

Figure 8. Synchronous detection. Extinction Cross-Section spectra obtained when all the target sequences are bound. 

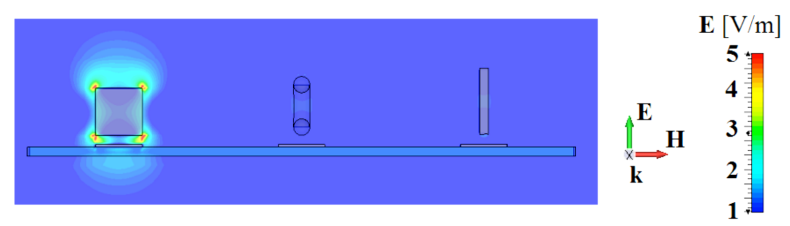

(a)
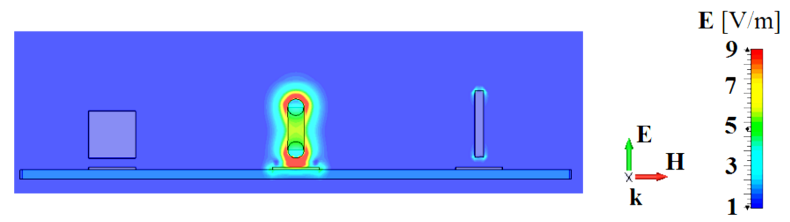

(b)
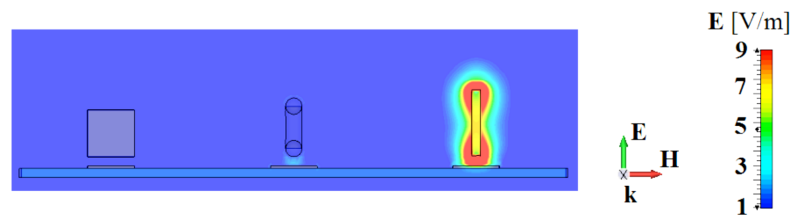

(c)

Figure 9. Near electric field distribution of the sensing platform at (a) the cube resonant wavelength $(542 \mathrm{~nm})$, (b) the rod resonant wavelength $(671 \mathrm{~nm})$, and (c) the elliptical cylinder resonant wavelength $(808 \mathrm{~nm})$. The incident electric field amplitude is $1 \mathrm{~V} / \mathrm{m}$.

alterations and then obtain the extinction-cross section spectra, exploiting the LSPR phenomenon. Synchronous and asynchronous nanoparticle transmissions are allowed, while a selective reception process occurs.

Future work will address the use of the proposed end-to-end model in other scenarios, such as drug delivery for innovative therapeutic and diagnostic applications.

\section{REFERENCES}

[1] I. F. Akyildiz and J. M. Jornet, "Electromagnetic Wireless Nanosensor Networks," Nano Communication Networks Elsevier Journal, Vol. 1, No. 1, 2010, pp. 3-19. http://dx.doi.org/10.1016/j.nancom.2010.04.001

[2] X. Wang, X. Lou, Y. Wang, Q. Guo, Z. Fang, X. Zhong, H. Mao, Q. Jin, L. Wu, H. Zhao and J. Zhao, "QDs-DNA Nanosensor for the Detection of Hepatitis b Virus DNA and the Single-Base Mutants," Biosensors and Bioelectronics, Vol. 25, No. 8, 2010, pp. 1934-1940. http://dx.doi.org/10.1016/j.bios.2010.01.007

[3] A. Abraham, R. Kannangai and G. Sridharan, "Nanotechnology: A New Frontier in Virus Detection in Clinical Practice," Indian Journal of Medical Microbiology, Vol. 26, No. 4, 2008, pp. 297-301. http://dx.doi.org/10.4103/0255-0857.43551

[4] V. Loscri, E. Natalizio, V. Mannara and G. Aloi, "A Novel Communication Technique for Nanobots Based on Acoustic Signals," In: Proceedings of the 7th International Conference on Bio-Inspired Models of Network, Information, and Computing Systems, ser. Bionetics'12, ICST, ICST (Institute for Computer Sciences, Social-In- formatics and Telecommunications Engineering), Brussels, 2012.

[5] L. La Spada, R. Iovine and L. Vegni, "Nanoparticle Electromagnetic Properties for Sensing Applications," Advances in Nanoparticles, Vol. 1, No. 2, 2012, pp. 9-14. http://dx.doi.org/10.4236/anp.2012.12002

[6] X. Qian, X. H. Peng, D. O. Ansari, Q. Yin-Goen, G. Z. Chen, D. M. Shin, L. Yang, A. N. Young, M. D. Wang and S. Nie, "In Vivo Tumor Targeting and Spectroscopic Detection with Surface-Enhanced Raman Nanoparticle Tags," Nature Biotechnology, Vol. 26, No. 1, 2008, pp. 83-90. http://dx.doi.org/10.1038/nbt1377

[7] J. Gao, X. Chen and Z. Cheng, "Near-Infrared Quantum Dots as Optical Probes for Tumor Imaging," Current Topics in Medicinal Chemistry, Vol. 10, No. 12, 2010, pp. 1147-1157. http://dx.doi.org/10.2174/156802610791384162

[8] S. Lian, P. Zhang, P. Gong, D. Hu, B. Shi, C. Zeng and L. Cai, "A Universal Quantum Dots-Aptamer Probe for Efficient Cancer Detection and Targeted Imaging," Current Topics in Medicinal Chemistry, Vol. 12, No. 10, 2012, pp. 7703-7708. http://dx.doi.org/10.1166/jnn.2012.6622

[9] C. Wang, X. Gao and X. Su, "In Vitro and in Vivo Imaging with Quantum Dots," Analytical and Bioanalytical Chemistry, Vol. 397, No. 4, 2010, pp. 1397-1415. http://dx.doi.org/10.1007/s00216-010-3481-6

[10] J. Jornet and I. Akyildiz, "Graphene-Based Nano-Antennas for Electromagnetic Nanocommunications in the Terahertz Band," 2010 Proceedings of the Fourth European Conference on Antennas and Propagation (EuCAP), Barcelona, 12-16 April 2010, pp. 1-5.

[11] G. W. Hanson, "Fundamental Transmitting Properties of Carbon Nanotube Antennas," IEEE Transactions on Antennas and Propagation, Vol. 53, No. 11, 2005, pp. 3426-3435. http://dx.doi.org/10.1109/TAP.2005.858865

[12] B. Atakan and O. Akan, "Carbon Nanotube-Based Nanoscale Ad Hoc Networks," IEEE Communications Magazine, Vol. 48, No. 6, 2010, pp. 129-135. http://dx.doi.org/10.1109/MCOM.2010.5473874

[13] NAD, "Nanoparticles and Nanotechnologies in Medicine," 2013. http://www.nadproject.eu/

[14] NANO3T. https://projects.imec.be/nano3t/

[15] PANOPTES. http://www.panoptesfp7.eu/

[16] I. F. Akyildiz, J. M. Jornet and M. Pierobon, "Nanonetworks: A New Frontier in Communications," Communications of the ACM, Vol. 54, No. 11, 2011, pp. 84-89. http://dx.doi.org/10.1145/2018396.2018417

[17] M. Pierobon and I. Akyildiz, "A Physical End-to-End Model for Molecular Communication in Nanonetworks," IEEE Journal on Selected Areas in Communications, Vol. 28, No. 4, 2010, pp. 602-611.

[18] B. Atakan, O. Akan and S. Balasubramaniam, "Body Area Nanonetworks with Molecular Communications in Nanomedicine," IEEE Communications Magazine, Vol. 50, No. 1, 2012, pp. 28-34. http://dx.doi.org/10.1109/MCOM.2012.6122529

[19] B. Atakan, S. Galmes and O. Akan, "Nanoscale Communication with Molecular Arrays in Nanonetworks," 
IEEE Transactions on NanoBioscience, Vol. 11, No. 2, 2012, pp. 149-160.

http://dx.doi.org/10.1109/TNB.2011.2181862

[20] T. Kobayashi, "Cancer Hyperthermia Using Magnetic Nanoparticles," Biotechnology Journal, Vol. 6, No. 11, 2011, pp. 1342-1347. http://dx.doi.org/10.1002/biot.201100045

[21] L. Kadouri, A. Hubert, Y. Rotenberg, T. Hamburger, M. Sagi, C. Nechushtan, D. Abeliovich and T. Peretz, "Cancer Risks in Carriers of the BRCA1/2 Ashkenazi Founder Mutations," Journal of Medical Genetics, Vol. 44, No. 7, 2007, pp. 467-471. http://dx.doi.org/10.1136/jmg.2006.048173

[22] D. Thompson, D. Easton and B. C. L. Consortium, "Cancer Incidence in BRCA1 Mutation Carriers," Journal of the National Cancer Institute, Vol. 94, No. 18, 2002, pp. 1358-1365. http://dx.doi.org/10.1093/jnci/94.18.1358

[23] L. Sun, C. Yu and J. Irudayaraj, "Raman Multiplexers for Alternative Gene Splicing," Analytical Chemistry, Vol. 80, No. 9, 2008, pp. 3342-3349. http://pubs.acs.org/doi/abs/10.1021/ac702542n

[24] M. A. Islam and H. Buschatz, "Mathematical Formulations of the Basic Diffusion Equation: A Discussion," BRAC University Journal, Vol. 1, No. 2, 2004, pp. 99107.

[25] E. Eckert and R. Drake, "Analysis of Heat and Mass Transfer," McGraw-Hill, New York, 1972.

[26] Y. Ali and L. Zhang, "Relativistic Heat Conduction," International Journal of Heat and Mass Transfer, Vol. 48, No. 12, 2005, pp. 2397-2406. http://dx.doi.org/10.1016/j.ijheatmasstransfer.2005.02.00 3

[27] A. Cavalcanti, B. Shirinzade, R. A. Freitas Jr. and T.
Hogg, "Nanorobot Architecture for Medical Target Identification," Nanotechnology, Vol. 19, No. 1, 2008, Article ID: 015103.

[28] K.-S. Lee and M. El-Sayed, "Gold and Silver Nanoparticles in Sensing and Imaging: Sensitivity of Plasmon Response to Size, Shape, and Metal Composition," Journal of Physical Chemistry B, Vol. 110, No. 39, 2006, pp. 19220-19225.

[29] A. Sihvola, "Electromagnetic Mixing Formulas and Applications," T. I. of Engineering and Technology, London, 2008.

[30] B. Gupta, W. Rouesnel, I. Y. Goon, R. Amal and J. J. Gooding, "DNA Hybridization for Nanocube Functionalization," International Conference on Nanoscience and Nanotechnology (ICONN), Sydney, 22-26 Febraury 2010, pp. 166-170.

[31] I. E. Sendroiu, M. E. Warner and R. M. Corn, "Fabrication of Silica-Coated Gold Nanorods Functionalized with DNA for Enhanced Surface Plasmon Resonance Imaging Biosensing Applications," Langmur, Vol. 25, No. 19, 2009, pp. 11282-11284.

[32] CST, "Microwave Studio 2012, Computer Simulation Technology," 2012. www.cst.com

[33] P. B. Johnson and R. W. Christy, "Optical Constants of the Noble Metals," Physical Review B, Vol. 6, No. 12, 1972, pp. 4370-4379.

[34] P. K. Jain, K. S. Lee, I. H. El-Sayed and A. El-Sayed, "Calculated Absorption and Scattering Properties of Gold Nanoparticles of Different Size, Shape, and Composition: Applications in Biological Imaging and Biomedicine," Journal of Physical Chemistry B, Vol. 110, No. 14, 2006, pp. 7238-7248. http://dx.doi.org/10.1021/jp057170o 